\title{
The Bruhat Order on the Involutions of the Symmetric Group
}

FEDERICO INCITTI*

incitti@mat.uniroma1.it

Dipartimento di Matematica, Università “La Sapienza”, P.le Aldo Moro 5, 00185, Rome, Italy

Received December 24, 2002; Revised September 23, 2003; Accepted October 3, 2003

\begin{abstract}
In this paper we study the partially ordered set of the involutions of the symmetric group $S_{n}$ with the order induced by the Bruhat order of $S_{n}$. We prove that this is a graded poset, with rank function given by the average of the number of inversions and the number of excedances, and that it is lexicographically shellable, hence Cohen-Macaulay, and Eulerian.
\end{abstract}

Keywords: symmetric group, Bruhat order, involution, $E L$-shellability, Cohen-Macaulay

\section{Introduction}

It is well-known that the symmetric group $S_{n}$ ordered by the Bruhat order encodes the cell decomposition of Schubert varieties (see, e.g., [8]). This partially ordered set has been studied extensively (see, e.g., [4, 5, 7, 11, 12, 19]) and it is known that it is a graded poset, with rank function given by the number of inversions, and that it is lexicographically shellable, hence Cohen-Macaulay, and Eulerian.

In [14] and [15] a vast generalization of this partial order has been considered, in relation to the cell decomposition of certain symmetric varieties. In this work we study this partial order in a special case that is particularly attractive from a combinatorial point of view, namely that of the involutions of $S_{n}$ with the order induced by the Bruhat order (see [14, Section 10]). Our main results are that this is a graded poset, with rank function given by the average of the number of inversions and the number of excedances, and that it is lexicographically shellable and Eulerian.

The organization of the paper is as follows. In Section 2 we give basic definitions, notation and results that will be needed later. Sections 3 and 4 are devoted to the study of some new combinatorial concepts, namely those of "suitable rise", "covering transformation" and "minimal covering transformation" of an involution, that play a crucial role in the sequel. In Sections 5, 6 and 7 we prove our main results, namely that the poset is graded, lexicographically shellable and Eulerian.

*This work is part of author's Ph.D. dissertation, written at "La Sapienza" University of Rome, under the direction of Professor Francesco Brenti. 


\section{Preliminaries}

In this section we recall some basic definitions, notation and results that will be used in the rest of this work.

We let $\mathbf{N}=\{1,2,3, \ldots\}$ and $\mathbf{Z}$ be the set of integers. For every $n \in \mathbf{N}$ we let $[n]=$ $\{1,2, \ldots, n\}$ and for every $n, m \in \mathbf{Z}$, with $n \leq m$, we let $[n, m]=\{n, n+1, \ldots, m\}$.

\subsection{Posets}

We follow [16, Chap. 3] for poset notation and terminology. In particular we denote by $\triangleleft$ the covering relation: $x \triangleleft y$ means that $x<y$ and there is no $z$ such that $x<z<y$. The Hasse diagram of a finite poset $P$ is the graph whose vertices are the elements of $P$, whose edges are the covering relations, and such that if $x<y$, then $y$ is drawn "above" $x$. A poset is said to be bounded if it has a minimum and a maximum, denoted by $\hat{0}$ and $\hat{1}$ respectively. For a bounded poset $P$ we denote by $\bar{P}$ the subposet $P \backslash\{\hat{0}, \hat{1}\}$. If $x, y \in P$, with $x \leq y$, we let $[x, y]=\{z \in P: x \leq z \leq y\}$, and we call it an interval of $P$. If $x, y \in P$, with $x<y$, a chain from $x$ to $y$ of length $k$ is a $(k+1)$-tuple $\left(x_{0}, x_{1}, \ldots, x_{k}\right)$ such that $x=x_{0}<x_{1}<\cdots<x_{k}=y$, denoted simply by " $x_{0}<x_{1}<\cdots<x_{k}$ ". A chain $x_{0}<x_{1}<\cdots<x_{k}$ is said to be saturated if all the relations in it are covering relations, and in this case we denote it simply by " $x_{0} \triangleleft x_{1} \triangleleft \cdots \triangleleft x_{k}$ ".

A poset is said to be graded of rank $n$ if it is finite, bounded and if all maximal chains of $P$ have the same length $n$. If $P$ is a graded poset of rank $n$, then there is a unique rank function $\rho: P \rightarrow[0, n]$ such that $\rho(\hat{0})=0, \rho(\hat{1})=n$ and $\rho(y)=\rho(x)+1$ whenever $y$ covers $x$ in $P$. Conversely, if $P$ is finite and bounded, and if such a function exists, then $P$ is graded of rank $n$.

If $P$ is a graded poset and $Q$ is a totally ordered set, an edge-labelling of $P$ with values in $Q$ is a function $\lambda:\left\{(x, y) \in P^{2}: x \triangleleft y\right\} \rightarrow Q$. If $\lambda$ is an edge-labelling of $P$, for every saturated chain $x_{0} \triangleleft x_{1} \triangleleft \cdots \triangleleft x_{k}$ we set

$$
\lambda\left(x_{0}, x_{1}, \ldots, x_{k}\right)=\left(\lambda\left(x_{0}, x_{1}\right), \lambda\left(x_{1}, x_{2}\right), \ldots, \lambda\left(x_{k-1}, x_{k}\right)\right) .
$$

An edge-labelling $\lambda$ of $P$ is said to be an EL-labelling if for every $x, y \in P$, with $x<y$, the following properties hold:

(1) there is exactly one saturated chain from $x$ to $y$, say $x=x_{0} \triangleleft x_{1} \triangleleft \cdots \triangleleft x_{k}=y$, such that $\lambda\left(x_{0}, x_{1}, \ldots, x_{k}\right)$ is a non-decreasing sequence (i.e., $\lambda\left(x_{0}, x_{1}\right) \leq \lambda\left(x_{1}, x_{2}\right) \leq \cdots \leq$ $\left.\lambda\left(x_{k-1}, x_{k}\right)\right)$;

(2) any other saturated chain from $x$ to $y$, say $x=y_{0} \triangleleft y_{1} \triangleleft \cdots \triangleleft y_{k}=y$, different from the previous one, is such that $\lambda\left(x_{0}, x_{1}, \ldots, x_{k}\right)<_{L} \lambda\left(y_{0}, y_{1}, \ldots, y_{k}\right)$, where $<_{L}$ denotes the lexicographic order $\left(\left(a_{1}, a_{2}, \ldots, a_{k}\right)<_{L}\left(b_{1}, b_{2}, \ldots, b_{k}\right)\right.$ if and only if $a_{i}<b_{i}$, where $\left.i=\min \left\{j \in[k]: a_{j} \neq b_{j}\right\}\right)$.

A graded poset $P$ is said to be lexicographically shellable, or EL-shellable, if it has an EL-labelling. 
Connections between $E L$-shellable posets and shellable complexes, Cohen-Macaulay complexes and Cohen-Macaulay rings can be found, for example, in [1-3, 9, 10, 13, 17]. Here we only recall some basic facts. The order complex $\Delta(P)$ of a poset $P$ is the simplicial complex of all chains of $P$. A poset $P$ is said to be shellable if $\Delta(P)$ is shellable, and Cohen-Macaulay if $\Delta(P)$ is Cohen-Macaulay (see, e.g., [3, Appendix], for the definitions of a shellable complex and of a Cohen-Macaulay complex). Furthermore, a poset is CohenMacaulay if and only if the Stanley-Reisner ring associated with it is Cohen-Macaulay (see, e.g., [13]). It is known that if a complex is shellable, then it is Cohen-Macaulay (see [9, Remark 5.3]). So the same holds for posets. Finally, Björner has proved the following (see $[3$, Theorem 2.3]).

Theorem 2.1 Let $P$ be a graded poset. If $P$ is EL-shellable then $P$ is shellable and hence Cohen-Macaulay.

A graded poset $P$ with rank function $\rho$ is said to be Eulerian if

$$
\mid\{z \in[x, y]: \rho(z) \text { is even }\}|=|\{z \in[x, y]: \rho(z) \text { is odd }\} \mid,
$$

for every $x, y \in P$ such that $x<y$.

In an $E L$-shellable poset there is a necessary and sufficient condition for the poset to be Eulerian. We state it in the following form (see [3, Theorem 2.7] and [18, Theorem 1.2] for proofs of more general results).

Theorem 2.2 Let $P$ be a graded EL-shellable poset and let $\lambda$ be an EL-labelling of $P$. Then $P$ is Eulerian if and only if for every $x, y \in P$ such that $x<y$, there is exactly one saturated chain from $x$ to $y$ with decreasing labels.

Finally, we refer to [10] or [17] for the definition of a Gorenstein poset, just recalling the following result (see, e.g., [17, Section 8]).

Theorem 2.3 Let $P$ be a graded Cohen-Macaulay poset. Then $P$ is Gorenstein if and only if the subposet of $P$ induced by $P \backslash\{x \in \bar{P}: x$ is comparable with every $y \in P\}$ is Eulerian.

\subsection{The Bruhat order on the symmetric group}

Given a set $T$ we let $S(T)$ be the set of all bijections $\pi: T \rightarrow T$. As ususal we denote by $S_{n}=S([n])$ the symmetric group, and we call its elements permutations.

If $\sigma \in S_{n}$ then we write $\sigma=\sigma_{1} \sigma_{2} \cdots \sigma_{n}$, to mean that $\sigma(i)=\sigma_{i}$ for every $i \in[n]$. We also write $\sigma$ in disjoint cycle form (see, e.g., [16, p. 17]), omitting to write the 1-cycles of $\sigma$. For example, if $\sigma=364152$, then we also write $\sigma=(1,3,4)(2,6)$. Given $\sigma, \tau \in S_{n}$, we let $\sigma \tau=\sigma \circ \tau$ (composition of functions) so that, for example, $(1,2)(2,3)=(1,2,3)$.

Given $\sigma \in S_{n}$, the diagram of $\sigma$ is a square of $n \times n$ cells, with the cell $(i, j)$ (i.e., the cell in column $i$ and row $j$, with the convention that the first column is the leftmost one and 
the first row is the lowest one) filled with a dot if and only if $\sigma(i)=j$. The diagonal of the diagram is the set of cells $\{(i, i): i \in[n]\}$.

Let $\sigma \in S_{n}$. As usual we denote by

$$
\operatorname{Inv}(\sigma)=\left\{(i, j) \in[n]^{2}: i<j, \sigma(i)>\sigma(j)\right\}
$$

the set of inversions of $\sigma$ and by inv $(\sigma)$ their number.

The (strong) Bruhat order of $S_{n}$ is the partial order relation on $S_{n}$, denoted by $\leq_{B}$, which is the transitive closure of the relation $\rightarrow$ defined by

$$
\begin{aligned}
\sigma \rightarrow \tau \Leftrightarrow & \text { there is a transposition }(i, j) \text { such that } \tau=\sigma(i, j) \\
& \text { and } \operatorname{inv}(\sigma) \leq \operatorname{inv}(\tau) .
\end{aligned}
$$

Let $\sigma \in S_{n}$. A rise of $\sigma$ is a pair $(i, j) \in[n]^{2}$ such that $i<j$ and $\sigma(i)<\sigma(j)$. A rise $(i, j)$ of $\sigma$ is said to be free if there is no $k \in[n]$ such that $i<k<j$ and $\sigma(i)<\sigma(k)<\sigma(j)$. The following is a well-known result.

Proposition 2.4 Let $\sigma, \tau \in S_{n}$. Then $\sigma \triangleleft \tau$ in the Bruhat order of $S_{n}$ if and only if $\tau=\sigma(i, j)$, for some free rise $(i, j)$ of $\sigma$.

Let $\sigma \in S_{n}$. For every $(h, k) \in[n]^{2}$ we set

$$
\sigma[h, k]=|\{i \in[h]: \sigma(i) \in[k, n]\}| .
$$

A fundamental characterization of the Bruhat order relation in $S_{n}$ is the following (see, e.g., [11]).

Proposition 2.5 Let $\sigma, \tau \in S_{n}$. Then $\sigma \leq_{B} \tau$ if and only if

$$
\sigma[h, k] \leq \tau[h, k]
$$

for every $(h, k) \in[n]^{2}$.

A consequence of Proposition 2.5 is the following.

Proposition 2.6 Let $\sigma, \tau \in S_{n}$. If $\sigma \leq_{B} \tau$ then $\sigma^{-1} \leq_{B} \tau^{-1}$.

We are interested in the set of involutions of $S_{n}$, which we denote by Invol(n). Note that a permutation is an involution if and only if its diagram is symmetric with respect to the diagonal. We wish to study the poset $\left(\operatorname{Invol}(n), \leq_{B}\right)$ of the involutions with the order induced by the Bruhat order of $S_{n}$. We will simply denote by $S_{n}$ and $\operatorname{Invol}(n)$ the respective posets with the Bruhat order.

In figures 1 and 2 are represented, respectively, the Hasse diagram of the poset $S_{4}$, with the involutions marked, and the Hasse diagram of the poset Invol(4). 


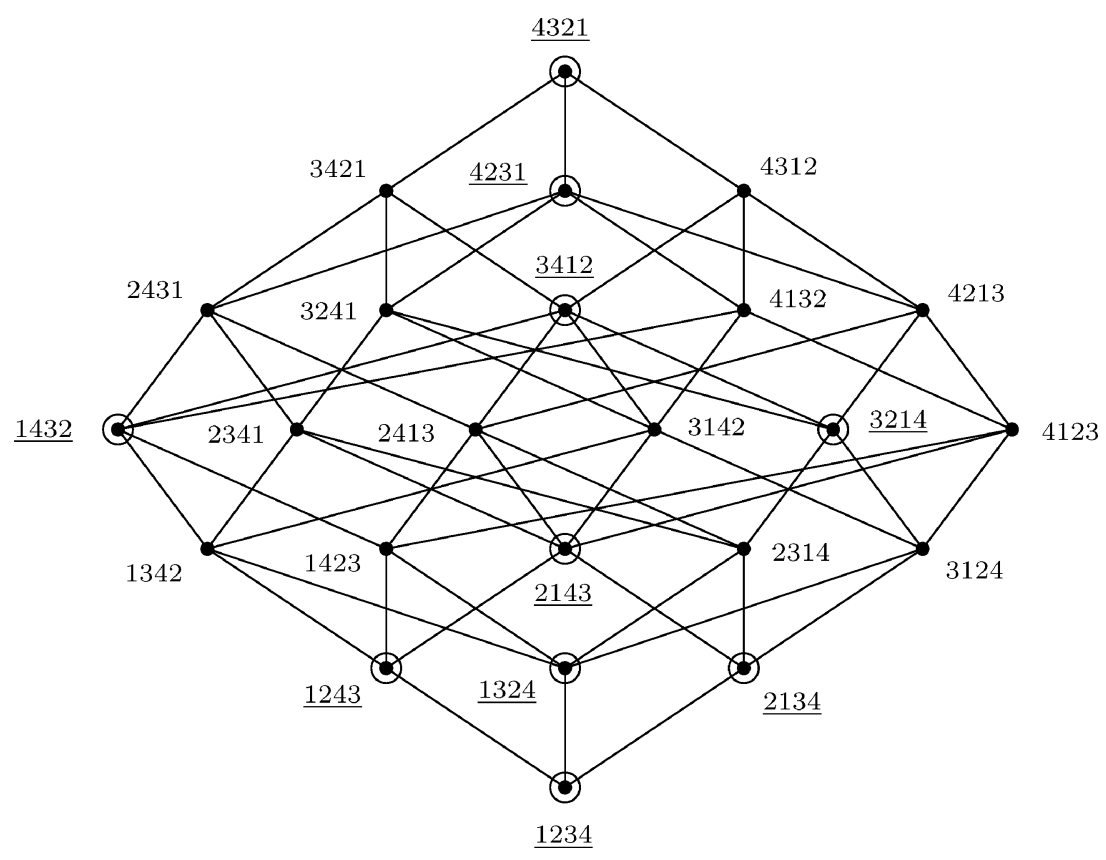

Figure 1. Hasse diagram of $S_{4}$.

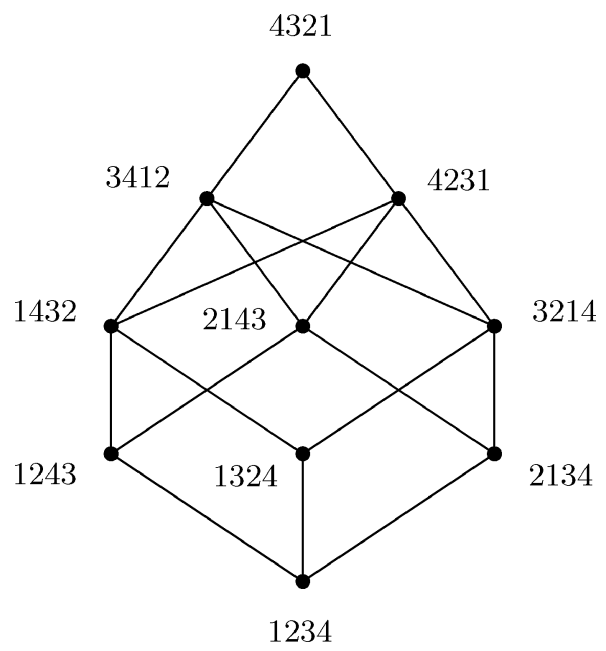

Figure 2. Hasse diagram of Invol(4). 
It is well-known that $S_{n}$ is a graded poset, with rank function given by the number of inversions, and that it is Eulerian (see, e.g., [19, p. 395]). It has been also shown that $S_{n}$ is $E L$-shellable (see, e.g., [7]). Our goal is to prove that similar results hold for the poset $\operatorname{Invol}(n)$.

It should be mentioned that the set of fixed-point-free involutions of $S_{n}$, partially ordered by the Bruhat order, has been recently studied in [6].

\section{Suitable rises and covering transformation}

In this section we introduce the concepts of "suitable rise" and "covering transformation" of an involution, which play a crucial role in the description of the covering relation in $\operatorname{Invol}(n)$

Let $\sigma \in S_{n}$. We denote by

$$
\begin{aligned}
I_{f}(\sigma) & =\operatorname{Fix}(\sigma)=\{i \in[n]: \sigma(i)=i\}, \\
I_{e}(\sigma) & =\operatorname{Exc}(\sigma)=\{i \in[n]: \sigma(i)>i\}, \\
I_{d}(\sigma) & =\operatorname{Def}(\sigma)=\{i \in[n]: \sigma(i)<i\},
\end{aligned}
$$

respectively the sets of fixed points, of excedances and of deficiencies of $\sigma$. As usual we denote by $\operatorname{exc}(\sigma)$ the number of excedances of $\sigma$.

The type of a rise $(i, j)$ is the pair $(a, b)$, where $a, b \in\{f, e, d\}$ are such that $i \in I_{a}(\sigma)$ and $j \in I_{b}(\sigma)$. A rise of type $(a, b)$ is also called an $a b$-rise. Furthermore, we need to distinguish between two kinds of $e e$-rises: an $e e$-rise $(i, j)$ is crossing if $i<\sigma(i)<j<\sigma(j)$, noncrossing if $i<j<\sigma(i)<\sigma(j)$. In other words, an $e e$-rise $(i, j)$ is crossing if the cells $(i, \sigma(j))$ and $(j, \sigma(i))$ are on opposite sides of the diagonal, and non-crossing otherwise.

For example, if $\sigma=321654$, the free rises of $\sigma$ are $(1,4),(1,5),(1,6),(2,4),(2,5)$, $(2,6),(3,4),(3,5),(3,6)$, whose types are, respectively, $(e, e),(e, f),(e, d),(f, e),(f, f)$, $(f, d),(d, e),(d, f),(d, d)$ (all nine possible types) and the ee-rise is crossing.

If $\sigma \in \operatorname{Invol}(n)$, then with every $e e$-rise of $\sigma$ is associated a symmetric $d d$-rise, with every $e f$-rise a $d f$-rise, with every $f e$-rise an $f d$-rise and with every $e d$-rise a $d e$-rise. This justifies the following definition.

Definition 3.1 Let $\sigma \in \operatorname{Invol}(n)$. A rise $(i, j)$ of $\sigma$ is suitable if it is free and if its type is one of the following: $(f, f),(f, e),(e, f),(e, e),(e, d)$.

We now introduce the main concept of this section.

Definition 3.2 Let $\sigma \in \operatorname{Invol}(n)$. Let $(i, j)$ be a suitable rise of $\sigma$. Depending on the type of $(i, j)$, we define a new involution obtained from $\sigma$, which we call covering transformation of $\sigma$ with respect to $(i, j)$, and denote by $c t_{(i, j)}(\sigma)$, as described in Table 1 .

The fourth column in Table 1 (with caption "Move") describes the action of $c t_{(i, j)}$ on the diagram of $\sigma$ : the black dots mark the positions of the elements of $\sigma$ which move in 
Table 1. Covering transformation.

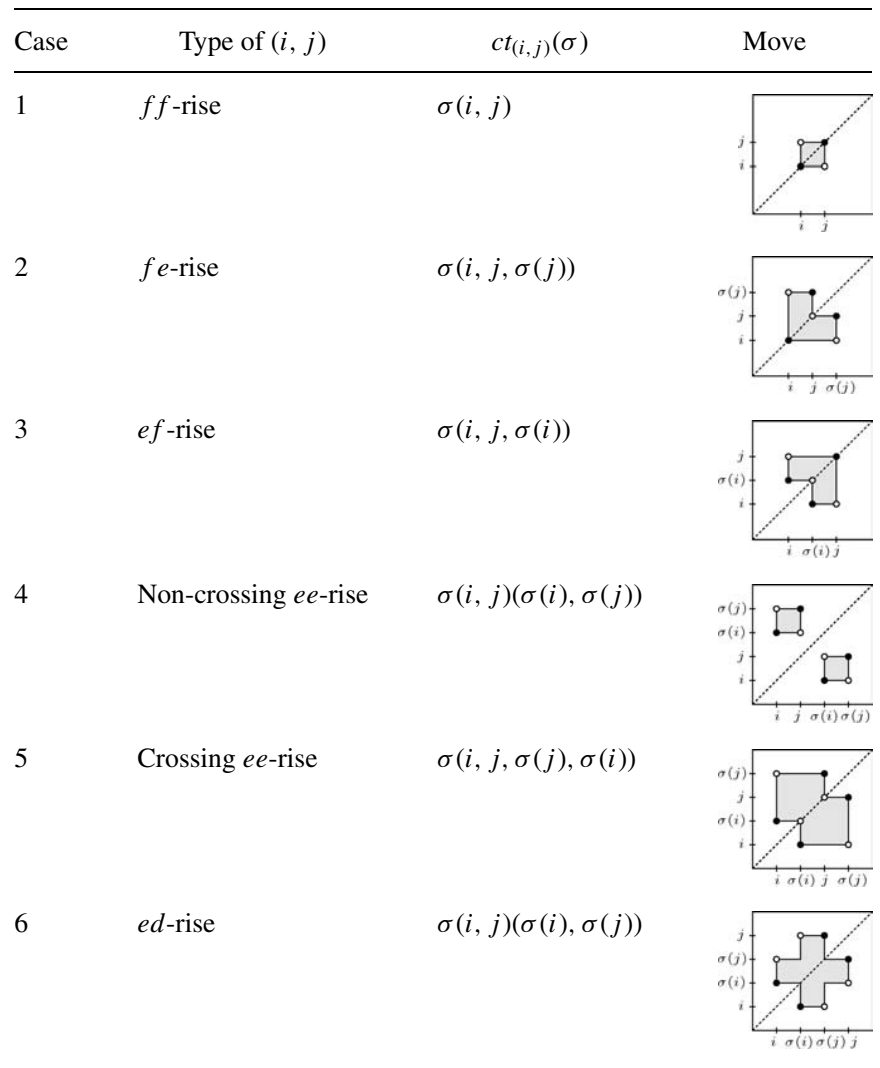

the transformation, while the white dots mark the positions of the new elements after the transformation. Inside the grey areas there are no other dots of $\sigma$. Note that in every case

$$
\left[\operatorname{ct}_{(i, j)}(\sigma)\right](i)=\sigma(j) .
$$

Lemma 3.3 Let $\sigma, \tau \in \operatorname{Invol}(n)$, with $\sigma \leq_{B} \tau$. Let $(i, j)$ be a suitable rise of $\sigma$, but not an ed-rise, such that $\sigma(i, j) \leq_{B} \tau$. Then

$$
c t_{(i, j)}(\sigma) \leq_{B} \tau .
$$

Proof: In case $1, c t_{(i, j)}(\sigma)=\sigma(i, j)$, and there is nothing to prove.

In all other cases (2,3, 4 and 5), we let $\sigma_{1}=\sigma(i, j), \sigma_{2}=\sigma_{1}^{-1}=\sigma(\sigma(i), \sigma(j))$ and $\sigma_{3}=c t_{(i, j)}(\sigma)$. We have $\sigma_{1} \leq_{B} \tau$ and, by Proposition 2.6, $\sigma_{2} \leq_{B} \tau$. We want to show that 
$\sigma_{3} \leq_{B} \tau$. In each of these four cases it's easy to check that for every $(h, k) \in[n]^{2}$ we have

$$
\sigma_{3}[h, k]= \begin{cases}\sigma_{1}[h, k], & \text { if } h>k, \\ \sigma_{2}[h, k], & \text { if } h \leq k .\end{cases}
$$

So, in every case, $\sigma_{3}[h, k] \leq \tau[h, k]$ and this implies $\sigma_{3} \leq_{B} \tau$.

It remains to consider case 6 ( $e d$-rise). Note that Lemma 3.3 does not hold in general if $(i, j)$ is a free $e d$-rise. For example, if we consider $\sigma=351624, \tau=653421$ and the free $e d$ rise $(1,6)$ of $\sigma$, we have $\sigma \leq_{B} \tau$ and $\sigma(1,6)=451623 \leq_{B} \tau$, but $c t_{(1,6)}(\sigma)=456123 \not \leq_{B} \tau$. We need to introduce a further new concept to handle $e d$-rises, and this is what we do in the next section.

\section{Minimal covering transformation}

In this section we introduce the concept of "minimal covering transformation" of an involution, crucial in the proofs that the poset $\operatorname{Invol}(n)$ is graded and $E L$-shellable.

Definition 4.1 Let $\sigma, \tau \in \operatorname{Invol}(n)$, with $\sigma \lesseqgtr B \quad \tau$. The difference index of $\sigma$ with respect to $\tau$, denoted by $d i_{\tau}(\sigma)$ (or simply $d i$ ), is the minimal index on which $\sigma$ and $\tau$ differ:

$$
d i_{\tau}(\sigma)=\min \{i \in[n]: \sigma(i) \neq \tau(i)\}
$$

Note that

$$
d i \leq \sigma(d i)<\tau(d i)
$$

To prove the two inequalities, first note that, by definition, $\sigma(d i) \neq \tau(d i)$. Since $\sigma$ and $\tau$ are involutions, they must differ at the index $\sigma(d i)$, and $d i$ is the minimal index at which they differ. Thus $d i \leq \sigma(d i)$. To prove the second inequality, suppose, to the contrary, that $\sigma(d i)>\tau(d i)$. In this case we would have $\sigma[d i, \sigma(d i)]=\tau[d i, \sigma(d i)]+1$. But $\sigma \leqq B \tau$ and, by Proposition 2.5, this implies $\sigma[d i, \sigma(d i)] \leq \tau[d i, \sigma(d i)]$, which is a contradiction. Thus $\sigma(d i)<\tau(d i)$.

Definition 4.2 Let $\sigma, \tau \in \operatorname{Invol}(n)$, with $\sigma \Varangle_{B} \tau$. The covering index of $\sigma$ with respect to $\tau$, denoted by $c i_{\tau}(\sigma)$ (or simply $c i$ ), is

$$
c i_{\tau}(\sigma)=\min \{j \in[d i+1, n]: \sigma(j) \in[\sigma(d i)+1, \tau(d i)]\} .
$$

To show that $c i$ is well-defined, set $k=\sigma(\tau(d i))$. If $k \in[d i-1]$, then $\sigma(k)=\tau(k)$, or equivalently $k=d i$, which is a contradiction. If $k=d i$, then $\sigma$ and $\tau$ agree at the index $d i$, which is also a contradiction. Thus $k \in[d i+1, n]$. Also, $\sigma(k)=\tau(d i)$, which implies that $c i$ is the minimum of a nonempty set.

By definition, $(d i, c i)$ is a free rise of $\sigma$, but more is true. 
Proposition 4.3 Let $\sigma, \tau \in \operatorname{Invol}(n)$, with $\sigma \lesseqgtr B \quad \tau$. Then $($ di, ci $)$ is a suitable rise of $\sigma$ and

$$
\sigma(d i, c i) \leq_{B} \tau
$$

Proof: We may assume, without loss of generality, that $d i=1$. As we have already observed, $d i$ is necessarily either a fixed point or an excedance of $\sigma$. So, for the first part, we only have to show that $(1, c i)$ is not an $f d$-rise. Suppose, to the contrary, that $(1, c i)$ is an $f d$-rise, so $\sigma(1)=1$ and $\sigma(c i)<c i$. By definition of $c i$, there is no $k \in[2, c i-1]$ such that $\sigma(k) \in[1, \tau(1)]$. In particular, since $\sigma(c i) \in[2, c i-1]$, we have $c i>\tau(1)$. Thus $|\{k \in[\tau(1)]: \sigma(k) \in[\tau(1)]\}|=1$, which implies

$$
\sigma[\tau(1), \tau(1)+1]=\tau(1)-1
$$

Also, since $d i$ is an excedance of $\tau$ and $\tau$ is an involution, we have $\mid\{k \in[\tau(1)]: \tau(k) \in$ $[\tau(1)]\} \mid \geq 2$, which implies

$$
\tau[\tau(1), \tau(1)+1] \leq \tau(1)-2 .
$$

So $\sigma[\tau(1), \tau(1)+1]>\tau[\tau(1), \tau(1)+1]$, but $\sigma \lesseqgtr B \quad \tau$ and, by Proposition 2.5 , this is a contradiction. Thus $(1, c i)$ is not an $f d$-rise.

For the second part, set $\chi=\sigma(1, c i)$ and $R=[1, c i-1] \times[\sigma(1)+1, \sigma(c i)]$. For every $(h, k) \in[n]^{2}$, we have

$$
\chi[h, k]= \begin{cases}\sigma[h, k]+1, & \text { if }(h, k) \in R, \\ \sigma[h, k], & \text { if }(h, k) \notin R .\end{cases}
$$

Thus, to prove that $\chi \leq_{B} \tau$, we only have to show that $\tau[h, k] \geq \sigma[h, k]+1$ for every $(h, k) \in R$. But if $(h, k) \in R$, then we have

$$
\sigma[h, k]=\sigma[h, \tau(1)+1] \leq \tau[h, \tau(1)+1] \leq \tau[h, k]-1,
$$

so $\chi \leq_{B} \tau$

Proposition 4.3 allows us to give the following definitions.

Definition 4.4 Let $\sigma, \tau \in \operatorname{Invol}(n)$, with $\sigma \lesseqgtr B \quad \tau$. The minimal covering rise of $\sigma$ with respect to $\tau$, denoted by $m c r_{\tau}(\sigma)$ (or simply $m c r$ ) is

$$
\operatorname{mcr}_{\tau}(\sigma)=(d i, c i) .
$$

The minimal covering transformation of $\sigma$ with respect to $\tau$, denoted by $\operatorname{mct}_{\tau}(\sigma)$ (or simply $m c t$ ) is the covering transformation of $\sigma$ with respect to the minimal covering rise:

$$
m c t_{\tau}(\sigma)=c t_{m c r}(\sigma)=c t_{(d i, c i)}(\sigma)
$$

We can now give our main result concerning ed-rises. 
Theorem 4.5 Let $\sigma, \tau \in \operatorname{Invol}(n)$, with $\sigma \Varangle_{B} \tau$. If $\operatorname{mcr}_{\tau}(\sigma)$ is an ed-rise then

$\operatorname{mct}_{\tau}(\sigma) \leq_{B} \tau$

Proof: We may assume, without loss of generality, that $d i=1$. Since $(d i, c i)$ is an $e d$-rise, and by the definitions of $d i$ and $c i$, we have that

$$
1<\sigma(1)<\sigma(c i) \leq \tau(1)<c i
$$

Set $a=\sigma(1), b=\sigma(c i), c=\tau(1)$ and $d=c i$, so $1<a<b \leq c<d$. Then

$$
m c t_{\tau}(\sigma)=\sigma(1, d)(a, b)
$$

Set $\chi=m c t_{\tau}(\sigma)$. We have $\sigma \leq_{B} \tau$ and we want to show that $\chi \leq_{B} \tau$. Let us consider the following subsets of $[n]^{2}$ :

$$
\begin{aligned}
& R_{1 a}=[1, a-1] \times[a+1, b], \quad R_{a 1}=[a, b-1] \times[2, a], \\
& R_{a a}=[a, b-1] \times[a+1, b], \\
& R_{b a}=[b, c-1] \times[a+1, b], \quad R_{a b}=[a, b-1] \times[b+1, c], \\
& R_{c a}=[c, d-1] \times[a+1, b], \quad R_{a c}=[a, b-1] \times[c+1, d], \\
& R_{\text {hor }}=[1, d-1] \times[a+1, c], \quad R_{\text {ver }}=[a, c-1] \times[2, d] .
\end{aligned}
$$

The situation is illustrated in figure 3, where the essential dots of the diagrams of $\sigma, \tau$ and $m c t_{\tau}(\sigma)$, and the sets just defined are represented. Note that, by the definition of $c i$, there are no dots of the diagram of $\sigma$ in $R_{h o r}$ and the only dot in $R_{v e r}$ is that in the cell $(b, d)$.

Also note that, for every $(h, k) \in[n]^{2}$, we have

$$
\chi[h, k]= \begin{cases}\sigma[h, k]+2, & \text { if }(h, k) \in R_{a a}, \\ \sigma[h, k]+1, & \text { if }(h, k) \in \bigcup_{x \in\{1, b, c\}} R_{x a} \cup \bigcup_{y \in\{1, b, c\}} R_{a y}, \\ \sigma[h, k], & \text { otherwise. }\end{cases}
$$

Furthermore, we have

$$
R_{a a} \subseteq R_{h o r} \cap R_{v e r}, \quad \bigcup_{x \in\{1, b, c\}} R_{x a} \subseteq R_{h o r}, \quad \bigcup_{y \in\{1, b, c\}} R_{a y} \subseteq R_{v e r} .
$$

Therefore, to prove that $\chi \leq_{B} \tau$, it suffices to show that $\tau[h, k] \geq \sigma[h, k]+2$ if $(h, k) \in$ $R_{\text {hor }} \cap R_{\text {ver }}$ and that $\tau[h, k] \geq \sigma[h, k]+1$ if $(h, k) \in R_{\text {hor }} \cup R_{\text {ver }}$.

Let $(h, k) \in R_{h o r}$. Then

$$
\sigma[h, c+1]=\sigma[h, k], \quad \tau[h, c+1] \leq \tau[h, k]-1 .
$$




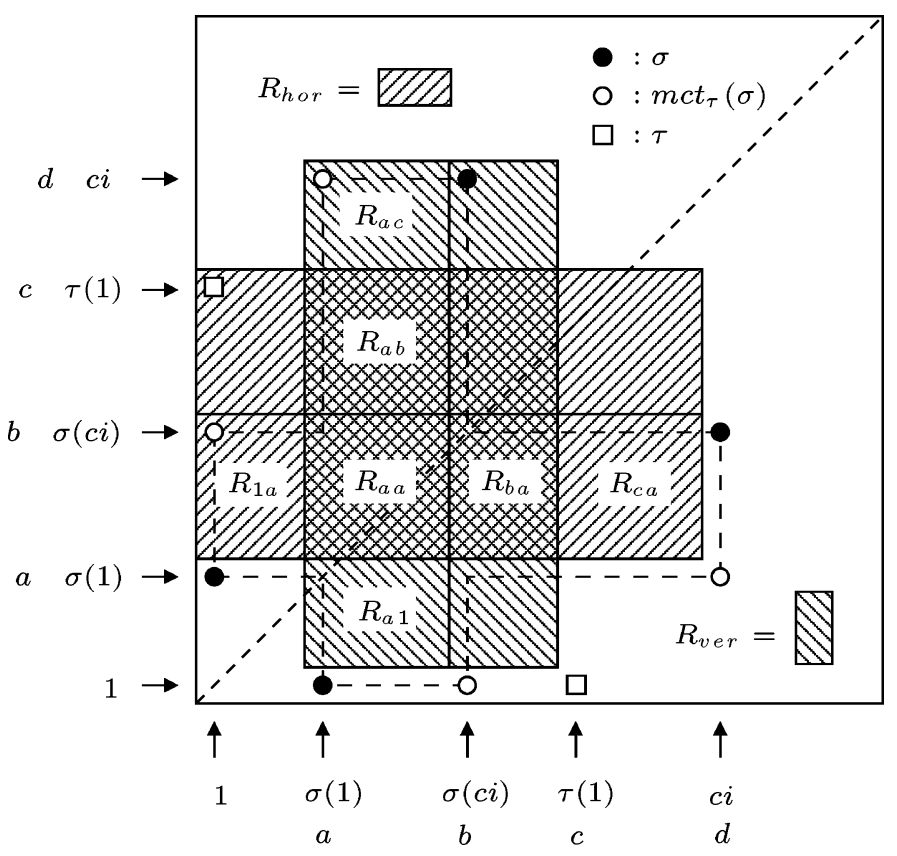

Figure 3. Proof of Theorem 4.5.

So, since $\sigma[h, c+1] \leq \tau[h, c+1]$, it follows that $\tau[h, k] \geq \sigma[h, k]+1$. Let $(h, k) \in R_{\text {ver }}$. Then

$$
\begin{aligned}
\sigma[c, k] & =\sigma[h, k]+c-h, \\
\tau[c, k] & =\tau[h, k]+|\{i \in[h+1, c-1]: \tau(i) \geq k\}| \\
& \leq \tau[h, k]+c-h-1 .
\end{aligned}
$$

So, since $\sigma[c, k] \leq \tau[c, k]$, it again follows that $\tau[h, k] \geq \sigma[h, k]+1$.

Finally, let $(h, k) \in R_{\text {hor }} \cap R_{\text {ver }}$. Then

$$
\begin{aligned}
& \sigma[c, c+1]=\sigma[h, k]+c-h, \\
& \tau[c, c+1] \leq \tau[c, k]-1 \leq \tau[h, k]+c-h-2 .
\end{aligned}
$$

So, since $\sigma[c, c+1] \leq \tau[c, c+1]$, we have $\tau[h, k] \geq \sigma[h, k]+2$.

The preceding result has the following important consequence.

Corollary 4.6 Let $\sigma, \tau \in \operatorname{Invol}(n)$, with $\sigma \lesseqgtr B$. Then

$$
m c t_{\tau}(\sigma) \leq_{B} \tau .
$$


Proof: If $m c r$ is an $e d$-rise, the result follows from Theorem 4.5. In any case, by Proposition 4.3, $(d i, c i)$ is a suitable rise of $\sigma$ such that $\sigma(d i, c i) \leq_{B} \tau$. So, if $m c r$ is not an $e d$-rise, the result follows from Lemma 3.3.

\section{5. $\operatorname{Invol}(n)$ is graded}

In this section we prove the first main result of this work, namely that $\operatorname{Invol}(n)$ is a graded poset, and we determine explicitly its rank function. In order to do this we first give a characterization of the covering relation in $\operatorname{Invol}(n)$, in terms of suitable rises and covering transformation.

Theorem 5.1 Let $\sigma, \tau \in \operatorname{Invol}(n)$. Then $\tau$ covers $\sigma$ in Invol $(n)$ if and only if $\tau=\operatorname{ct}_{(i, j)}(\sigma)$, for some suitable rise $(i, j)$ of $\sigma$.

Proof: Let $\tau=c t_{(i, j)}(\sigma)$, for some suitable rise $(i, j)$ of $\sigma$. Let us examine all the six possible cases (see Table 1). In case $1, \tau=\sigma(i, j)$ covers $\sigma$ in $S_{n}$ so in particular in $\operatorname{Invol}(n)$. In case $2, \tau=\sigma(i, j)(j, \sigma(j))$; in case $3, \tau=\sigma(i, j)(j, \sigma(i))$; in cases 4 and $6, \tau=\sigma(i, j)(\sigma(i), \sigma(j))$. In all these four cases, $\tau$ is greater than $\sigma$ in the Bruhat order, and has distance 2 from it in the Hasse diagram of $S_{n}$. The permutations in the interval $[\sigma, \tau]$ of $S_{n}$ covering $\sigma$ cannot be involutions, so $\tau$ covers $\sigma$ in $\operatorname{Invol}(n)$. Finally, in case 5, $\tau=\sigma(i, j)(j, \sigma(j))(\sigma(j), \sigma(i))$ is greater than $\sigma$ in the Bruhat order and has distance 3 from it in the Hasse diagram of $S_{n}$. In this case the permutations in the interval $[\sigma, \tau]$ of $S_{n}$ covering $\sigma$, and those covered by $\tau$ cannot be involutions, so again $\tau$ covers $\sigma$ in $\operatorname{Invol}(n)$.

On the other hand, if $\tau \neq c t_{(i, j)}(\sigma)$ for every suitable rise $(i, j)$ of $\sigma$, then, by Corollary 4.6, we have

$$
\sigma \varliminf_{B} m c t_{\tau}(\sigma) ફ_{B} \tau .
$$

Thus $\tau$ does not cover $\sigma$ in $\operatorname{Invol}(n)$.

As an example of application of Theorem 5.1, consider $\sigma=321654 \in \operatorname{Invol}(6)$. The suitable rises of $\sigma$ are $(1,4),(1,5),(1,6),(2,4)$ and $(2,5)$, and we have $c t_{(1,4)}(\sigma)=623451$, $c t_{(1,5)}(\sigma)=523614, c t_{(1,6)}(\sigma)=426153, c t_{(2,4)}(\sigma)=361452$ and $c t_{(2,5)}(\sigma)=351624$. So

$$
\{\tau \in \operatorname{Invol}(n): \sigma \triangleleft \tau \text { in } \operatorname{Invol}(n)\}=\{623451,523614,426153,361452,351624\} .
$$

We can now state and prove the main result of this section.

Theorem 5.2 The poset Invol( $n)$ is graded, with rank function $\rho$ given by

$$
\rho(\sigma)=\frac{\operatorname{inv}(\sigma)+\operatorname{exc}(\sigma)}{2}
$$


for every $\sigma \in \operatorname{Invol}(n)$. In particular Invol(n) has rank

$$
\rho(\operatorname{Invol}(n))=\left\lfloor\frac{n^{2}}{4}\right\rfloor .
$$

Proof: By Theorem 5.5, it suffices to show that

$$
\rho\left(c t_{(i, j)}(\sigma)\right)=\rho(\sigma)+1
$$

for every $\sigma \in \operatorname{Invol}(n)$ and for every suitable rise $(i, j)$ of $\sigma$.

As we have already seen in the proof of Theorem 5.1, the increase in the number of inversions going from $\sigma$ to $c t_{(i, j)}(\sigma)$ is 1 in case 1 , it is 2 in cases $2,3,4,6$, and it is 3 in case 5. On the other hand in case 1 the involution $c t_{(i, j)}(\sigma)$ has one excedance more than $\sigma$, in cases 2, 3, 4, 6 the number of excedances does not change, and in case 5 the involution $\operatorname{ct}_{(i, j)}(\sigma)$ has one excedance less than $\sigma$. Therefore, in each case $\rho$ increases by 1 .

For the second part, it suffices to observe that the maximum of $S_{n}$, which is also the maximum of $\operatorname{Invol}(n)$, has $\lfloor n / 2\rfloor$ excedances and $n(n-1) / 2$ inversions.

\section{6. $\operatorname{Invol}(n)$ is $E L$-shellable}

In this section we prove that the poset $\operatorname{Invol}(n)$ is $E L$-shellable, defining a particular edgelabelling of $\operatorname{Invol}(n)$, which we call "standard", and showing that it is an $E L$-labelling.

Theorem 5.1 allows us to give the following definition.

Definition 6.1 The standard edge-labelling of Invol( $n)$, with values in the set $\{(i, j) \in$ $\left.[n]^{2}: i<j\right\}$ (totally ordered by the lexicographic order), is defined in the following way: for every $\sigma, \tau \in \operatorname{Invol}(n)$ such that $\tau$ covers $\sigma$ in $\operatorname{Invol}(n)$, if $(i, j)$ is the suitable rise of $\sigma$ such that $\tau=c t_{(i, j)}(\sigma)$, then we set

$$
\lambda(\sigma, \tau)=(i, j) .
$$

We can now prove the $E L$-shellability of $\operatorname{Invol}(n)$.

Theorem 6.2 The poset Invol(n) is EL-shellable, having the standard edge-labelling as an EL-labelling.

Proof: Suppose we label the edges of the Hasse diagram of $\operatorname{Invol}(n)$ with the standard labelling.

Let $\sigma, \tau \in \operatorname{Invol}(n)$, with $\sigma \lesseqgtr B \tau$. Consider the saturated chain from $\sigma$ to $\tau$

$$
\sigma=\sigma_{0} \triangleleft \sigma_{1} \triangleleft \cdots \triangleleft \sigma_{k}=\tau,
$$


defined by

$$
\sigma_{i}=m c t_{\tau}\left(\sigma_{i-1}\right)
$$

for every $i \in[k]$. Corollary 4.6 ensures that $\sigma_{i} \leq_{B} \tau$ for every $i \in[k-1]$.

By the definitions of $m c t, d i$ and $c i$, this chain has, among all the saturated chains from $\sigma$ to $\tau$, the minimal labelling in the lexicographic order.

We now prove that it has increasing labels. Suppose, by contradiction, that at a certain step there is a decrease in the labels. We may assume, without loss of generality, that this happens at the first step. Thus

$$
\sigma_{1}=m c t_{\tau}(\sigma)=c t_{(d i, c i)}(\sigma)
$$

and

$$
\sigma_{2}=c t_{(i, j)}\left(\sigma_{1}\right)
$$

with $(i, j)<_{L}(d i, c i)$. So either $i<d i$ or $i=d i$ and $j<c i$. If $i<d i$, since $\sigma$ and $\tau$ must differ at the index $i$, the minimality of $d i$ is contradicted. If $i=d i$ and $j<c i$, since $\sigma(j) \in[\sigma(d i)+1, \tau(d i)]$, the minimality of $c i$ is contradicted.

It remains to show that any other saturated chain from $\sigma$ to $\tau$, different from the previuos one, has at least one decrease. Let

$$
\sigma=\tau_{0} \triangleleft \tau_{1} \triangleleft \cdots \triangleleft \tau_{k}=\tau
$$

be such a saturated chain. Set $h=\min \left\{i \in[k-1]: \tau_{i} \neq \sigma_{i}\right\}, d i=d i_{\tau}\left(\sigma_{h-1}\right)$ and $c i=c i_{\tau}\left(\sigma_{h-1}\right)$. So

$$
\sigma_{h}=m c t_{\tau}\left(\sigma_{h-1}\right)=c t_{(d i, c i)}\left(\sigma_{h-1}\right)
$$

and

$$
\tau_{h}=c t_{(i, j)}\left(\sigma_{h-1}\right),
$$

for some suitable rise $(i, j)$ of $\sigma_{h-1}$ different from $(d i, c i)$ and lexicographically greater than it. So either $d i<i$ or $d i=i$ and $c i<j$.

If $d i<i$, then in the covering relations $\tau_{h} \triangleleft \tau_{h+1} \triangleleft \cdots \triangleleft \tau_{k}=\tau$ there must be at least one with label containing $d i$, so lower than $(i, j)$.

Suppose $d i=i$ and $c i<j$. Since the dot in column $d i$ has to move from row $\sigma(d i)$ to row $\tau(d i)$ and because of the presence in the diagram of $\sigma$ of the dot in the cell $(c i, \sigma(c i))$, in the covering relations $\tau_{h} \triangleleft \tau_{h+1} \triangleleft \cdots \triangleleft \tau_{k}=\tau$ either there is one with label ( $\left.d i, c i\right)$, so lower than $(i, j)$, or there is one with label starting with $c i$, followed by one with label starting with $d i$, so again with a decrease.

As a consequence, by Theorem 2.1, we have the following.

Corollary 6.3 The poset Invol(n) is Cohen-Macaulay. 


\section{7. $\operatorname{Invol}(n)$ is Eulerian}

In this section we prove that the poset $\operatorname{Invol}(n)$ is Eulerian. In order to do this, we introduce some notions which, in some sense, invert those introduced in Sections 3 and 4.

Definition 7.1 Let $\tau \in \operatorname{Invol}(n)$. An inversion $(i, j)$ of $\tau$ is inv-suitable if $(i, j)$ is a suitable rise of some $\sigma \in \operatorname{Invol}(n)$ and $c t_{(i, j)}(\sigma)=\tau$. We call such a $\sigma$ (obviously unique) the inverse covering transformation of $\tau$ with respect to $(i, j)$ and we denote it by $i c t_{(i, j)}(\tau)$.

Obviously $i c t_{(i, j)}\left(c t_{(i, j)}(\sigma)\right)=\sigma$ and $c t_{(i, j)}\left(i c t_{(i, j)}(\tau)\right)=\tau$. We summarize the action of the inverse covering transformation on the diagram of an involution in Table 2, with a notation similar to that used in Table 1 . In this case the black dots denote $\tau$ and the white dots denote $i c t_{(i, j)}(\tau)$.

Definition 7.2 Let $\sigma, \tau \in \operatorname{Invol}(n)$, with $\sigma \lesseqgtr B \quad \tau$. The minimal covering inversion of $\tau$ with respect to $\sigma$, denoted by $m c i_{\sigma}(\tau)$ (or simply $m c i$ ), is the minimal (in the lexicographic order) inv-suitable inversion $(i, j)$ of $\tau$ such that $\sigma \leq_{B} i c t_{(i, j)}(\tau)$.

Table 2. Inverse covering transformation.

\begin{tabular}{ll}
\hline Case & Move \\
\hline 1 &
\end{tabular}

2

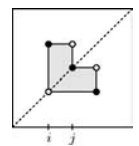

3

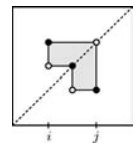

4

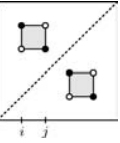

5

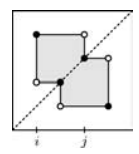

6

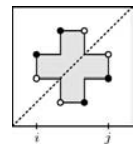


The minimal inverse covering tranformation of $\tau$ with respect to $\sigma$, denoted by $\operatorname{mict}_{\sigma}(\tau)$ (or simply mict), is the inverse covering transformation of $\tau$ with respect to the minimal covering inversion:

$$
\operatorname{mict}_{\sigma}(\tau)=i c t_{m c i}(\tau)
$$

We can now prove that the condition of Theorem 2.2 holds for the poset $\operatorname{Invol}(n)$, and thus that it is Eulerian.

Theorem 7.3 The poset Invol( $n)$ is Eulerian.

Proof: Suppose we label the edges of the Hasse diagram of $\operatorname{Invol}(n)$ with the standard labelling.

Let $\sigma, \tau \in \operatorname{Invol}(n)$, with $\sigma \oiint_{B} \tau$. By Theorem 2.2, we only have to show that there is exactly one saturated chain from $\sigma$ to $\tau$ with decreasing labels.

In this proof we use the following terminology: if $(i, j)$ is an inv-suitable inversion of $\tau$ we call it simply a move for $\tau$, precisely an $a$-move, with $a \in$ [6], if we are in case $a$ of Table 2. Furthermore, if $i c t_{(i, j)}(\tau)=\sigma$, then we write

$$
\tau \underset{(i, j)}{\triangleright} \sigma
$$

We divide the proof in two parts. We first prove that there is at least one saturated chain from $\sigma$ to $\tau$ with decreasing labels. Consider the descending chain

$$
\tau=\sigma_{0} \triangleright \sigma_{1} \triangleright \cdots \triangleright \sigma_{k}=\sigma
$$

defined by

$$
\sigma_{i}=\operatorname{mict}_{\sigma}\left(\sigma_{i-1}\right)
$$

for every $i \in[k]$. We claim that it has increasing labels (so the corresponding ascending chain will have decreasing labels). Suppose, by contradiction, that at a certain step there is a decrease in the labels. We may assume, without loss of generality, that this happens at the first step. So

$$
\sigma_{0} \underset{(i, j)}{\triangleright} \sigma_{1} \underset{\left(i^{\prime}, j^{\prime}\right)}{\triangleright} \sigma_{2}
$$

with $\left(i^{\prime}, j^{\prime}\right)<_{L}(i, j)$. There are two cases: either $i^{\prime}<i$ or $i^{\prime}=i$ and $j^{\prime}<j$.

If $i^{\prime}<i$, then $(i, j)$ cannot be the minimal choice for $\sigma_{0}$, since $\sigma_{0}$ must have an inv-suitable inversion containing $i^{\prime}$. This contradicts the definition of the chain.

If $i=i^{\prime}$ and $j^{\prime}<j$, again $(i, j)$ cannot be the minimal choice for $\sigma_{0}$. The proof of this fact is a case-by-case verification, depending on the type of $(i, j)$. We show some cases, leaving the others to the reader. 


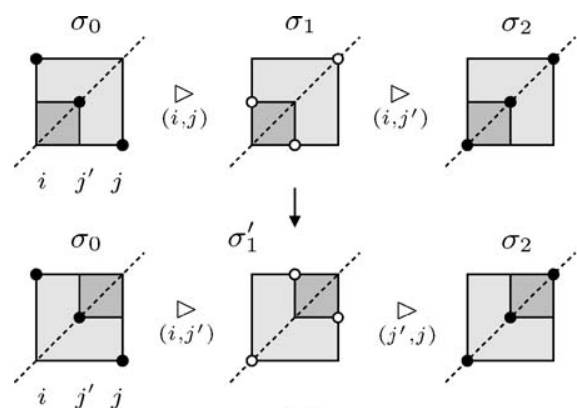

(a)

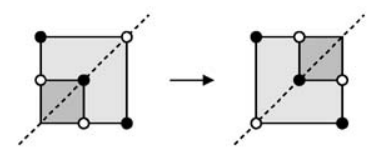

(b)

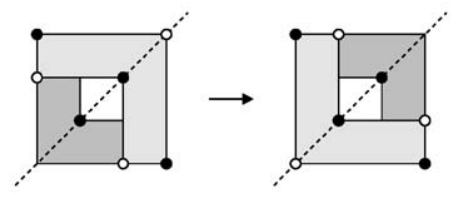

(c)

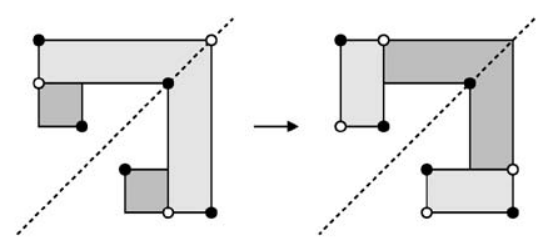

(e)

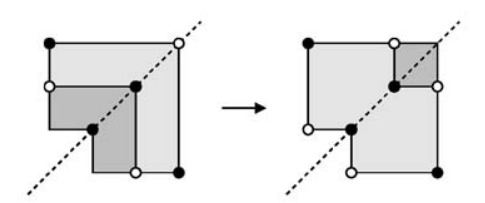

(d)

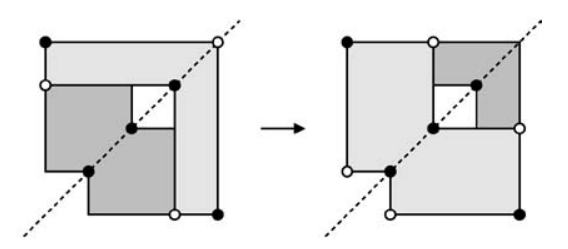

(f)

Figure 4. Proof of Theorem 7.3.

First of all note that $(i, j)$ cannot be a 1-move or a 2-move, in fact in this case we could not apply to $\sigma_{1}$ a move $\left(i, j^{\prime}\right)$, with $j^{\prime}<j$. If $(i, j)$ is a 3 -move and $\left(i, j^{\prime}\right)$ is a 1 -move, the situation is illustrated in figure 4(a): if we apply to $\sigma_{0}$ the two moves $\left(i, j^{\prime}\right)$ and $\left(j^{\prime}, j\right)$ (in this order), we again reach $\sigma_{2}$ :

$$
\sigma_{0} \underset{\left(i, j^{\prime}\right)}{\triangleright} \sigma_{1}^{\prime} \underset{\left(j^{\prime}, j\right)}{\triangleright} \sigma_{2}
$$

But $\left(i, j^{\prime}\right)<_{L}(i, j)$, so $(i, j)$ is not the minimal choice for $\sigma_{0}$. In the picture we represent a pair of moves by colouring the areas "enclosed" in the moves, with a lighter grey for the first move and a darker grey for the second one; the arrow represents the possibility of substituting a pair of moves with another pair reaching the same involution. Figure 4(b) is the synthetic version of figure 4(a). If $(i, j)$ is a 3-move, all other cases are synthetically described in figure 4(c-f), with the notation described above. If $(i, j)$ is a 4,5 or 6 -move the reasoning is similar. In each case we get a contradiction. 
We now prove that any other saturated chain from $\sigma$ to $\tau$, different from the previous one, has at least one increase. Let

$$
\tau=\tau_{0} \triangleright \tau_{1} \triangleright \cdots \triangleright \tau_{k}=\sigma
$$

be a saturated descending chain from $\tau$ to $\sigma$, different from the previous one. We will prove that in it there is at least one decrease (so the corresponding ascending chain will have at least one increase). Set $h=\min \left\{i \in[k-1]: \tau_{i} \neq \sigma_{i}\right\}$ and $m c i_{\sigma}\left(\sigma_{h-1}\right)=(i, j)$. We have

$$
\sigma_{h}=i c t_{(i, j)}\left(\sigma_{h-1}\right)
$$

and

$$
\tau_{h}=i c t_{\left(i^{\prime}, j^{\prime}\right)}\left(\sigma_{h-1}\right)
$$

for some inv-suitable inversion $\left(i^{\prime}, j^{\prime}\right)$ of $\sigma_{h-1}$ different from $(i, j)$ and lexicographically greater than it. So either $i<i^{\prime}$ or $i=i^{\prime}$ and $j<j^{\prime}$.

If $i<i^{\prime}$, then in the covering relations $\tau_{h} \triangleright \tau_{h+1} \triangleright \cdots \triangleright \tau_{k}=\sigma$ there must be one with label containing $i$, so lower than $\left(i^{\prime}, j^{\prime}\right)$.

Suppose $i=i^{\prime}$ and $j<j^{\prime}$. We want to show that in the labelling of the descending chain $\sigma_{h-1} \triangleright \tau_{h} \triangleright \tau_{h+1} \triangleright \cdots \triangleright \tau_{k}=\sigma$ there is at least one decrease. Suppose, by contradiction, that it has increasing labels. If $l=\min \left\{s \in[h, k]: \tau_{s}(i)=\sigma(i)\right\}$, then

$$
\sigma_{h-1} \underset{\left(i, j^{\prime}\right)}{\triangleright} \tau_{h} \underset{\left(i, j_{1}\right)}{\triangleright} \tau_{h+1} \underset{\left(i, j_{2}\right)}{\triangleright} \cdots \triangleright \tau_{l-1} \underset{\left(i, j_{l-h}\right)}{\triangleright} \tau_{l},
$$

with $(j<) j^{\prime}<j_{1}<j_{2}<\cdots<j_{l-h}$. We want to show that this is in contradiction with the fact that $(i, j)$ is an inv-suitable inversion of $\sigma_{h-1}$. The proof of this fact is again a case-by-case verification, depending on the type of $(i, j)$.

If $(i, j)$ is a 1 -move, a 3 -move or a 6-move, then the $\left(i, j_{r}\right)$ 's can only be 6-moves, and none of these can send the dot in column $i$ on or below row $\sigma_{h}(i)$. But $\sigma(i) \leq \sigma_{h}(i)$, and we have a contradiction.

If $(i, j)$ is a 2-move, a 4-move or a 5-move, then the sequence of the $\left(i, j_{r}\right)$ 's can only be realized by a sequence (possibly empty) of 4-moves, possibly followed by a 3 or a 5-move (but not both) and then by a sequence (possibly empty) of 6-moves. If $(i, j)$ is a 4-move, then none of these moves can send the dot in column $i$ on or below row $\sigma_{h}(i)$. If $(i, j)$ is a 2 -move or a 5 -move, then the 3 or 5 -move is the only one that can move the dot in column $i$ on row $j$, and in this case none of the following 6-moves can move that dot on or below row $\sigma_{h}(i)$. But, as before, $\sigma(i) \leq \sigma_{h}(i)$, so in each case we get a contradiction.

Furthermore, by Theorem 2.3, we can conclude the following.

Corollary 7.4 The poset Invol( $n)$ is Gorenstein. 


\section{References}

1. K. Baclawski, "Cohen-Macaulay ordered sets," J. Algebra 63 (1980), 226-258.

2. A. Björner, A.M. Garsia, and R.P. Stanley, An Introduction to Cohen-Macaulay Partially Ordered Sets, Ordered Sets (Banff., Alta., 1981), pp. 583-615.

3. A. Björner, "Shellable and Cohen-Macaulay partially ordered sets," Trans. Amer. Math. Soc. 260 (1980), 159-183.

4. A. Björner and M. Wachs, "Bruhat order of Coxeter groups and shellability," Adv. in Math. 43 (1982), 87-100

5. V.V. Deodhar, "Some characterizations of Bruhat ordering on a Coxeter group and determination of the relative Möbius function," Invent. Math. 39(2) (1977), 187-198.

6. R.S. Deodhar and M.K. Srinivasan, "A statistic on involutions," J. Alg. Combin. 13(2) (2001), 187-198.

7. P.H. Edelman, "The Bruhat order of the symmetric group is lexicographically shellable," Proc. Amer. Math. Soc. 82 (1981), 355-358.

8. W. Fulton, "Young tableaux," With Applications to Representation Theory and Geometry, London Mathematical Society Student Texts, 35, Cambridge University Press, Cambridge, 1997.

9. A.M. Garsia, "Combinatorial methods in the theory of Cohen-Macaulay rings," Adv. in Math. 38(3) (1980), 229-266.

10. M. Hochster, Cohen-Macaulay Rings, Combinatorics, and Simplicial Complexes, Ring Theory II (Proc. Second Conf. Univ. Oklahoma, 1975), pp. 171-223.

11. R.A. Proctor, "Classical Bruhat orders and lexicographic shellability," J. Algebra 77 (1982), 104-126.

12. N. Reading, "Order dimension, strong Bruhat order and lattice properties for posets," Order 19 (2002), 73-100.

13. G. Reisner, "Cohen-Macaulay quotients of polynomial rings," Adv. in Math. 21 (1976), 30-49.

14. R.W. Richardson and T.A. Springer, "The Bruhat order on symmetric varieties," Geom. Dedicata 35(1-3) (1990), 389-436.

15. R.W. Richardson and T.A. Springer, "Complements to: The Bruhat order on symmetric varieties," Geom. Dedicata 49(2) (1994), 231-238.

16. R.P. Stanley, Enumerative Combinatorics, Vol. 1, Wadsworth and Brooks/Cole, Pacific Grove, CA, 1986.

17. R.P. Stanley, "Cohen-Macaulay complexes," Higher Combinatorics (Proc. NATO Advanced Study Inst., Berlin, 1976), pp. 51-62.

18. R.P. Stanley, "Supersolvable lattices," Algebra Universalis 2 (1972), 197-217.

19. D.N. Verma, "Möbius inversion for the Bruhat ordering on a Weyl group," Ann. Sci. École Norm. Sup. 4 (1971), 393-398. 